\title{
Statyba
}

\section{MULTI-PURPOSE SELECTONOVATION OF ALTERNATIVE VARIANTS OF TECHNOLOGICAL DECISIONS IN BUILDING}

\section{E. K. Zavadskas}

To cite this article: E. K. Zavadskas (1996) MULTI-PURPOSE SELECTONOVATION OF ALTERNATIVE VARIANTS OF TECHNOLOGICAL DECISIONS IN BUILDING, Statyba, 2:5, 58-63, DOI: $10.1080 / 13921525.1996 .10531548$

To link to this article: https://doi.org/10.1080/13921525.1996.10531548

Published online: 26 Jul 2012.

Submit your article to this journal $₫$

Џ Article views: 51 


\section{МНОГОЦЕЛЕВАЯ СЕЛЕКТОНОВАЦИЯ ПРОГНОЗНЫХ ВАРИАНТОВ ТЕХНОЛОГИЧЕСКИХ РЕШЕНИЙ СТРОИТЕЛЬНОГО ПРОИЗВОДСТВА}

\section{Э.К. Завадскас}

\section{1. Введене. Принциы многоцелевой селектоновяция}

Многоцелевая селектоновация (МСH) [1] прогнозных вариантов проводится с учетом принпипов сложности, многоцелевости, многоаспектности, комплексности, многоэтапности, мнотовариантности, слабоструктуризованности и поликритериапности.

Сложность МСН определяется сложностью структуры и недостаточным уровнем разработки понятий. Сложность структуры вырахается в том, тто МСН постоянно видоизменяется в зависимости от изучаемого строительного объекта или конкретного технологического процесса. Это объективный и закономерный процесс.

МСН явяется многоцелевой, так как учитывает множество целей хозяйства, явцищегося сложной иерархической соџиаљно-экономической системой.

Проблемы МСН отличаюся многоаспектностыю - стремлением достичь требуемого или желаемого уровня решением целого ряда задач технического, информащионного, социалыного, правового, математического характера.

Комплексность проблемы МСН обусловливается необходимостью решения разнородньх задач с целыю воздействия на составляошие элементы производственного процесса строительства (средства и предметы труда, трудовые, материальные, энергетические и информационные ресурсы) для получения продухшии требуемого качества в заданные сроки.

Комплексность проблем, решаемкх МСН, подтверждается и тем, что технологичность решений определяется на всех стадиях проектирования, подготовки производства и вышолнения производственных процессов. Отсюда вытекают свойства интердисциплинарности и дедуктивности МСН как раздела научной дисциплины технологии строительного производства. Интердисцилинарность заключается в использовании разгичных областей знаний (инженерньх наук, математики, экономики и т.д.).

Дедуктивность МСН проявляется в привлечении научных дисциплин, оценивающих эффективность принимаемых строительных решений в каком-нибудь одном аспекте, и объединении их в единое целое.

Следует отметить, что хотя конечные цели проектировщиков и производственников, принадлежаших к разным уровням системы технической подтотовки строительства, явцяотся обшими, однако текуцие подцели каждого участника этого процесса не всегда строго совпадают. Поэтому в результате неполного совпадепия целей и требований подсистем различного уровня усложняется исследование проблем МСН и создание эффективных методов их решения.

Поскольку МСН приходится осуществлять на разных этапах проектирования и подготовки строительного производства [2, 3], для проблем МСН характерен принцип многоэтапности. МСН 


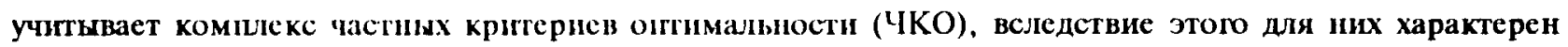
принци поликритернальости. ЧКО (иолџимснсиональне показатели эффективности - ППЭ) имеют, как правило, разний физический смысл и разные дименсии (размерности), поэтому для проблем МСН характерны свойства полидименсионалынсти ЧКО. ЧКО (ППЭ) долхиы быть релевантными, т.е. бнть точными, своевременными и обладать достаточно полюой информацией.

Технология строгтельног производства является многовариантиой, поэтому при рсшении проблем МСН имеет место принцип многовариантности. Кроме того, для МСН характерно свойство изоформизма, характеризуюшее аналогичност этапов и методов, применемьх при решении конкретньх задач разработки, оценки и вжбора техиологий строительното производства для различнх типов зданий и сооружении, выполнения отдельных видов конструкщй и технологических процессов.

Задачи МСН при разработке принциииальо новых перспективньх технологически решенин строительного производства для тех случаев, когда отсутствуют результать экспериментальньх исследованй, а имеюшиеся ЧКО (ППЭ), полученные на основании результатов предварительньх расчетов, отличаются значительной степенью неопределенности, могут быть решены с использованием критериев теории итр $[2,4,5]$ или методом определения функция полезности на мнохестве многофакторньх вариантов [6].

\section{2. Принцишы многоцелевой селектоноващи прогнозных вариантов}

При МСН прогнозншх вариантов с учетом научно-технического прогресса необходимо учитыпать условия решения крупнњх экономических и социашыњх задач на анализируемом этапе. Условия решенпя этіх задач связаны с постановкой и решением целого комглекса тахах предварительнхх частних задач, как комплексное изучение целей развития отрасли, сощияљноэкономических, научно-технических задач с учетом постоянио меняошихся условий строительного производства, Ігривязки прогнозируемого перспективного технологического решения $\mathbf{x}$ внешней среде, согласования и взаимоувязки прогнозируемьх параметров. Окончательным этапом МСН прогнозных вариантов является комплексная оценка альтернатив развития и вњбор рационашното варианта из множества возможных альтернатив на основе хомплекса ЧКО (ППЭ).

При таком подходе обеспечивается одновременное прогнозирование по ряду важнейших показателей эффективности. На основе приниипа многовариантности могут быть получены различные затраты трудовьх, материальны и энергетических ресурсов, а также разтичные стонмостные похазатели, различные показатели продолжительности выполнения строительньх процессов и качества получаемой продукции. Поэтому предлагаемые альтернативы разработки перспективнх технологиеских решений строительного производства необходимо оценивать и окончательный выбор осушествлть с тем условием, чтобы прииттый ращиональный вариант в пределах доверительной области удовлетворял многоцелевому принцину.

Принятое перспективное технологическое решение должно удовлетворять одновременно хозяйствеппым интересам и игтересам строгтельной организации. В связи с этим выбор перспективнхх решений догжен быть иаправлен одновременио на максимизацию ввода, минимзацюо пронзводственных затрат при условии усигения режима экономии за счет ращнонаного использования трудовкх, энергетических и материальньх ресурсов.

Такмм образом, оценка прогнозных вариантов должна быть поликритериальной и учитьвающей постоянное совершенствование технологических решений на базе комплекса ЧКО (ППЭ), наиболее полно характеризуюших ix эффективность. 


\section{3. Методнка репения задач многоцелевой селектоновапни прогнозных варнантов}

Решение задач $\mathrm{MCH}$ прогнозных вариантов возможно с использованием рассмотренных ранее методов МСН [1-6], объединенных в универсальную интерактивную систему поддержки принятия решений [2]. Эффективность прогнозных вариантов при этом оценивается с учетом единства натуральных и стоимостных ЧКО (ППЭ). МСН осуществляется с учетом фактора времени и в общем виде может быть представлена в виде выражения:

$$
\begin{gathered}
\operatorname{opt}\left[\bar{x}\left(t_{z}\right), q\left(t_{z}\right)\right] \equiv \max _{i}\left[\left\{\bar{x}_{j}\left(t_{z}\right)\right\} /\left\{q_{j}\left(t_{z}\right)\right\}\right] ; \\
\forall i j z ; \quad i=\overline{1, m} ; \quad j=\overline{1, n} ; \quad z=\overline{1, l} ; \\
\sum_{j=1}^{n} q_{j}\left(t_{z}\right)=1,
\end{gathered}
$$

где $\bar{x}\left(t_{z}\right)=\left\{\bar{x}_{1}\left(t_{z}\right), \bar{x}_{2}\left(t_{z}\right), \ldots, \bar{x}_{j}\left(t_{z}\right), \ldots, \bar{x}_{n}\left(t_{z}\right)\right\}-$ вектор ЧКО (ППЭ), представляющих собой техникоэкономические показатели и качественные характеристики [14] в нормализованном виде в момент прогнозируемого периода $t$;

$q\left(t_{z}\right)=\left\{q_{1}\left(t_{z}\right), q_{2}\left(t_{z}\right), \ldots, q_{j}\left(t_{z}\right), \ldots, q_{n}\left(t_{z}\right)\right\}-$ вехтор показателей весомости (значимости) ЧКО (ППЭ) в момент прогнозируемого периода $t_{z}$, определяемый с помощью экспертншх оценок.

На основании исходных данных (учитываемьх ЧКО) за определенный исследуемњй период с помощыю различнбх методов прогнозирования [7-12] получены $m$ прогнознбх вариантов технологических решений строительного производства, в результате чего строится исходная матрища $P=\left[x_{j i z}\right]$ (табл. 1).

С целью МСН ЧКО (ППЭ) сравниваемњх аљьтернатив $i(i=\overline{1, m})$ нормализуются с использованием методов, рассмотренншх в [2]. В результате строится нормализованная матрица $\bar{P}=\left[\bar{x}_{j i z}\right]$.

Дия определения показателей весомости ЧКО (ППЭ) прогнозируемнх вариантов рекомендуется использовать метод парньх сравнений [2]. Следует отметить, что в разные годн прогнозируемого периода вектор значений $q$ может быть разичным.

МСН из множества возможных методов рекомендуется осуществлять по приншипу относительной уступки (критерию $K^{O У}$ )

$$
\begin{gathered}
K_{i z}^{\mathrm{OY}} \equiv \operatorname{opt}\left[\bar{x}\left(t_{z}\right), q\left(t_{z}\right)\right] \equiv \max _{i} \prod_{j=1}^{n} \bar{x}_{j}\left(t_{z}\right)^{q_{j}\left(t_{z}\right)} \\
\forall i j z ; \quad i=\overline{1, m} ; \quad j=\overline{1, n} ; \quad z=\overline{1, l}
\end{gathered}
$$

или по критерию близости к идеальной точке [14]

$$
\begin{gathered}
\max K_{\text {Бит } i z}=\frac{L_{i z}^{-}}{L_{i z}^{+}+L_{i z}^{-}} ; \\
i=\overline{1, m} ; \quad z=\overline{1, l}
\end{gathered}
$$

где $K_{\text {Битіz }}-$ критерий близости $\mathbf{x}$ идеальной точке прогнозируемого варианта $i$ в год прогнозируемого периода $z$;

$L_{i z}^{+}-$расстояние между каждым и идеальным вариантами в год $\mathrm{z}$ прогнозируемого периода; 


\section{$L_{i z}^{-}-$расстояние между каждым и негативно-идеальным вариантами в год $z$}

прогнозируемого периода.

На основании решения по выражениям (2) и (3) вычисляются бездименсиональные (безразмернже) оценки критериев $\mathrm{MCH}$ для отдельных вариантов в определенный год $z$ прогнозируемого периода (табл. 2, 3).

Таблица 1. Варианты прогнозных технологических решений строитељього производства

\begin{tabular}{|c|c|c|c|c|c|c|c|}
\hline \multirow{2}{*}{$\begin{array}{c}\text { पKO } \\
\text { (ППЭ) } \\
j\end{array}$} & \multirow{2}{*}{$\begin{array}{c}\text { No } \\
\text { варианта } \\
i\end{array}$} & \multicolumn{6}{|c|}{ Годы прогнозируемого периода } \\
\hline & & $t_{1}$ & $t_{2}$ & $\ldots$ & $t_{z}$ & $\ldots$ & $t_{1}$ \\
\hline \multirow[t]{6}{*}{$x_{1}$} & $B_{1}$ & $x_{111}$ & $x_{112}$ & $\cdots$ & $x_{11 z}$ & $\cdots$ & $x_{11 l}$ \\
\hline & $B_{2}$ & $x_{121}$ & $x_{122}$ & $\cdots$ & $x_{12 z}$ & $\cdots$ & $x_{12 l}$ \\
\hline & $\vdots$ & $\vdots$ & $\vdots$ & $:::$ & $\vdots$ & $:::$ & $\vdots$ \\
\hline & $B_{i}$ & $x_{1 i 1}$ & $x_{1 i 2}$ & $\ldots$ & $x_{1 i z}$ & $\cdots$ & $x_{1 i l}$ \\
\hline & $\vdots$ & $\vdots$ & $\vdots$ & $:: \vdots$ & $\vdots$ & $:::$ & $\vdots$ \\
\hline & $B_{m}$ & $x_{1 m 1}$ & $x_{1 m 2}$ & $\ldots$ & $x_{1 m z}$ & $\ldots$ & $x_{1 m l}$ \\
\hline \multirow[t]{6}{*}{$x_{2}$} & $B_{1}$ & $x_{211}$ & $x_{212}$ & $\cdots$ & $x_{21 z}$ & $\cdots$ & $x_{21 l}$ \\
\hline & $B_{2}$ & $x_{221}$ & $x_{222}$ & $\cdots$ & $x_{222}$ & $\cdots$ & $x_{22 l}$ \\
\hline & $\vdots$ & $\vdots$ & $\vdots$ & $:::$ & $\vdots$ & $:::$ & $\vdots$ \\
\hline & $B_{i}$ & $x_{2 i 1}$ & $x_{2 i 2}$ & $\ldots$ & $x_{2 i z}$ & $\cdots$ & $x_{2 i l}$ \\
\hline & $\vdots$ & $\vdots$ & $\vdots$ & $:::$ & $\vdots$ & :: & $\vdots$ \\
\hline & $B_{m}$ & $x_{2 m 1}$ & $x_{2 m 2}$ & $\ldots$ & $x_{2 m z}$ & $\ldots$ & $x_{2 m l}$ \\
\hline & $\vdots$ & & & & & & \\
\hline \multirow[t]{7}{*}{$x_{j}$} & $B_{1}$ & $x_{j 11}$ & $x_{j 12}$ & $\ldots$ & $x_{j 1 z}$ & $\ldots$ & $x_{j l l}$ \\
\hline & $B_{2}$ & $x_{J 21}$ & $x_{j 22}$ & $\cdots$ & $x_{j 2 z}$ & $\cdots$ & $x_{j 2 l}$ \\
\hline & $\vdots$ & $\vdots$ & $\vdots$ & $:::$ & $\vdots$ & $:: \vdots$ & $\vdots$ \\
\hline & $B_{i}$ & $x_{j i 1}$ & $x_{j i 2}$ & $\ldots$ & $x_{j i z}$ & $\ldots$ & $x_{j i l}$ \\
\hline & $\vdots$ & $\vdots$ & $\vdots$ & $:::$ & $\vdots$ & $:: \vdots$ & $\vdots$ \\
\hline & $B_{m}$ & $x_{j m 1}$ & $x_{\jmath m 2}$ & $\ldots$ & $x_{j m z}$ & $\ldots$ & $x_{j m l}$ \\
\hline & $\vdots$ & & & & & & \\
\hline \multirow[t]{6}{*}{$x_{n}$} & $B_{1}$ & $x_{n 11}$ & $x_{n 12}$ & $\ldots$ & $x_{n 1 z}$ & $\ldots$ & $x_{n 1 l}$ \\
\hline & $B_{2}$ & $x_{n 21}$ & $x_{n 22}$ & $\ldots$ & $x_{n 2 z}$ & $\cdots$ & $x_{n 2 l}$ \\
\hline & $\vdots$ & $\vdots$ & $\vdots$ & $: \because:$ & $\vdots$ & $:::$ & $\vdots$ \\
\hline & $B_{i}$ & $x_{n i 1}$ & $x_{n 2}$ & $\ldots$ & $x_{n i z}$ & $\ldots$ & $x_{n i l}$ \\
\hline & $\vdots$ & $\vdots$ & $\vdots$ & $:::$ & $\vdots$ & $:: \vdots$ & $\vdots$ \\
\hline & $B_{m}$ & $x_{n m 1}$ & $x_{n m 2}$ & $\ldots$ & $x_{n m z}$ & $\ldots$ & $x_{n m l}$ \\
\hline
\end{tabular}


Таблища 2. Оценки критерия МСН прогнозных вариантов технологических решений строительнго производства $K^{\text {OY }}$

\begin{tabular}{|c|c|c|c|c|c|c|}
\hline \multirow{2}{*}{$\begin{array}{c}\text { Прогнозный } \\
\text { вариант развития } \\
\text { технологического } \\
\text { решення }\end{array}$} & \multicolumn{6}{|c|}{ Годы прогнозируемого периода } \\
\hline & $t_{1}$ & $t_{2}$ & ... & $t_{z}$ & $\ldots$ & $t_{l}$ \\
\hline 1 & $K_{11}^{O y}$ & $K_{12}^{\mathrm{OY}}$ & $\cdots$ & $K_{12}^{\mathrm{O} J}$ & $\ldots$ & $K_{11}^{\mathrm{Oy}}$ \\
\hline 2 & $K_{21}^{O y}$ & $K_{22}^{\mathrm{Oy}}$ & $\ldots$ & $K_{22}^{O}$ & $\ldots$ & $K_{2 l}^{\mathrm{OY}}$ \\
\hline$\vdots$ & $\vdots$ & $\vdots$ & $\vdots: \vdots$ & $\vdots$ & $\vdots \vdots$ & $\vdots$ \\
\hline$i$ & $K_{i 1}^{\text {Oy }}$ & $K_{i 2}^{\mathrm{OY}}$ & $\cdots$ & $K_{i z}^{\mathrm{O} J}$ & $\cdots$ & $K_{i I}^{\mathrm{OY}}$ \\
\hline$\vdots$ & $\vdots$ & $\vdots$ & $\vdots: \vdots$ & $\vdots$ & $\vdots:$ & $\vdots$ \\
\hline$m$ & $K_{m 1}^{O Y}$ & $K_{m 2}^{O y}$ & $\ldots$ & $K_{m z}^{O J}$ & $\ldots$ & $K_{m l}^{O y}$ \\
\hline
\end{tabular}

Таблща 3. Оценки критерия $\mathrm{MCH}$ прогнозных вариантов технологических решгений строитељного производства $K_{\text {Бит }}$

\begin{tabular}{|c|c|c|c|c|c|c|}
\hline \multirow{2}{*}{$\begin{array}{c}\text { Прогнозный } \\
\text { вариант развития } \\
\text { технологического } \\
\text { решення }\end{array}$} & \multicolumn{6}{|c|}{ Годы прогнозируемого периода } \\
\hline & $t_{1}$ & $t_{2}$ & $\ldots$ & $t_{\tau}$ & $\ldots$ & $t_{l}$ \\
\hline 1 & $K_{\text {Бит 11 }}$ & $K_{\text {Бит 12 }}$ & $\cdots$ & $K_{\text {Бит } 1 z}$ & $\cdots$ & $K_{\text {Бит } 1}$ \\
\hline 2 & $K_{\text {Бит } 21}$ & $K_{\text {Бит 22 }}$ & $\cdots$ & $K_{\text {Бит 2z }}$ & $\cdots$ & $K_{\text {Бит } 2 l}$ \\
\hline$\vdots$ & $\vdots$ & $\vdots$ & $\vdots: \vdots$ & $\vdots$ & $\because::$ & $\vdots$ \\
\hline$i$ & $K_{\text {Бнті1 }}$ & $K_{\text {Бит } 12}$ & $\cdots$ & $K_{\text {Бит } i z}$ & $\cdots$ & $K_{\text {Бит } i l}$ \\
\hline$\vdots$ & $\vdots$ & $\vdots$ & $\dddot{\vdots}:$ & $\vdots$ & $:::$ & $\vdots$ \\
\hline$m$ & $K_{\text {Бит } m 1}$ & $K_{\text {Бит } m 2}$ & $\cdots$ & $K_{\text {Бит } m z}$ & $\cdots$ & $K_{\text {Бит } m l}$ \\
\hline
\end{tabular}

Используя результаты табл. 2, определяется рациональный с учетом развития всех прогнозируемых ЧКО (ППЭ) вариант разработки или более широкого внедрения перспективного технологического решения строительного производства. Кроме того, строятся ряды предпочтительности применения прогнозируемьх вариантов на отдельные годы прогнозируемого периода.

$$
\begin{gathered}
\left\{\vec{B}_{t_{z}}\right\}=\left\{B_{1} \succ B_{2} \succ \ldots \succ B_{m}\right\}, \\
\forall i z ; \quad i=\overline{1, m} ; \quad z=\overline{1, l} .
\end{gathered}
$$

Ряды предпочтительности при возможном количестве перестановок $\pi=m$ ! используются как основа дальейших перспективных расчетов.

\section{4. Заключение}

Многоцелевая селектоновация вариантов развития перспективньх технологическх решений строительного производства позволяет определить наиболее рациональный по комплексу оцениваемьх частных критериев оптимальности (полидименсиональньх показателей эффективности) и таким образом способствует одновременному достижению нескольких конечных целей. 


\section{ЛЕтература}

1. Э.К.Завацскас. Многоцелевая селектоновация технологических решений строительного производства (теоретические основы). Вильнюс, 1988. $108 \mathrm{c}$.

2. Э.К.Завадскас. Системотехническая оценка технологических решений строительного производства. Ленинград: Стройиздат, Ленинградское отделение, 1991. 257 с.

3. E.K.Zavadskas. Mehrzielselektierung bei der Bauvorbereitung // Statyba. N 3(3). Vilnius: Technika, 1995, p. 58-80.

4. F.Peldschus. Zur Anwendung der Theorie der Spiele für Aufgaben der Bautechnologie. Diss. B. TH Leipzig, 1986. $119 \mathrm{~S}$.

5. F.Peldschus, E.Vaigauskas, E.Zavadskas. Technologische Entscheidungen bei der Berücksichtigung mehrerer Ziele. Berlin: Bauplanung-Bautechnik, 1983, N 4, S. 173-175.

6. E.KZavadskas. Variantenauswahl mit der Nutzensfunktion // Wissenschaftliche Zeitschrift der Technische Hochschule Leipzig, 1990, Jg. 14, H. 5/6, S. 263-273.

7. В.Г.Гмопинский. Инхенерное прогнозирование. М.: Энергоиздат, 1982. 208 с.

8. В.Г.Дерзский и др. Прогнозирование технихо-экономических параметров новой техники. Киев: Науковя думка, 1982. $176 \mathrm{c}$

9. В.А.Іанцов. Прогнозирование эффективности механизации. Ленинград: Стройнздат, $1982.176 \mathrm{c.}$

10. Рабочая книта по прогнозированию. М:Мысть, $1982.430 \mathrm{c}$.

11. Дж.Мартино. Технологическое прогнозирование: Пер. с англ. М.: Прогресс, 1977. 592 с.

12. Б.Мельникас, Э.Завадскас. Применение математических методов при решении некоторых задач разработки технологии строительного производства. Вильнос, 1980. $118 \mathrm{c.}$

13. I.Löhne, F.Peldschus, E.Zavadskas. LEVI - ein Programm zur Lösung von Mehrkriteriellen Entscheidungsaufgaben // Statybos technologija ir menedžmentas, N 7. Vilnius: Technika, 1993, p. 8-14.

14. Э.К.Завадскас. Обобщенная системотехническая оценка и определение предшочтителыности проектных решений в строительстве // Statyba, N 4(4). Vilnius: Technika, 1995, p. 31-39.

Iteikta 19960215

\section{TECHNOLOGINIU SPRENDIMU STATYBOJE PROGNOZUOJAMU VARIANTU DAUGLATIKSLE SELEKTONOVACIJA}

\section{E.KZavadskas}

Santrauka

Nagrinejjami pagrindiniai daugiatikslès selektonovacijos metodologiniai principai. Sie principai pritaikomi kuriant prognozuojamy technologinį sprendimy statyboje daugiatikslio ịvertinimo metodiką. Pasiūlytas bendras algoritmas ir du varianty vertinimo kriterijai - santykinès nuolaidos ir artumo idealiam taškui. Turint paskaičiuotas kriteriju reikšmes rikiuojamos prognozuojamy variantu prioritety eilutès atskiriems prognozuojamo periodo metams.

\section{MULTI-PURPOSE SELECTONOVATION OF ALTERNATIVE VARIANTS OF TECHNOLOGICAL DECISIONS IN BUILDING}

\section{E.K.Zavadskas}

\section{Su m mary}

The main methodological principles of multi-purpose selectonovation are discussed in the paper. These principles are applied to the development of methods for multi-purpose evaluation of alternative technological desicions in building. A common algorithm and two evaluation criteria are suggested: the relative concession criterion and the criterion of proximity to an ideal point. The values of criteria having been estimated, the rows of alternative variant priorities for the definite years of future period are arranged. 